\title{
Characterizing Health Care Utilization, Direct Costs, and Comorbidities Associated with Interstitial Cystitis: A Retrospective Claims Analysis
}

\author{
Amy Tung, PharmD, MS; Zsolt Hepp, PharmD, MS; Aasthaa Bansal, PhD; \\ and Emily Beth Devine, PhD, PharmD, MBA
}

\begin{abstract}
BACKGROUND: Interstitial cystitis (IC) is a debilitating condition that affects up to $5 \%$ of the U.S. population. This condition is characterized by bladder pain, urinary urgency and frequency, nocturia, and, in some patients, bladder lesions called Hunner's lesions (HL). IC patients who have HL experience a clinical course that is distinct from those without $\mathrm{HL}$ and, as a result, respond differently to existing treatments. Without effective and lasting therapeutic options, IC patients are expected to experience a reduced quality of life and be a significant economic burden. Previous research describing the burden of IC is not only outdated but lacks stratification by HL.
\end{abstract}

OBJECTIVES: To (a) characterize health care utilization, direct costs, and comorbidities associated with IC and (b) elucidate differences between patients with and without $\mathrm{HL}$.

METHODS: A retrospective analysis was conducted using health care claims from the Truven Health MarketScan Research Databases. Adults with an incident IC diagnosis between 2009 and 2014 were identified and matched 1:4 to non-IC patients on age, gender, and geographic region. Health care utilization, direct costs, and comorbidities during the first 12 months after diagnosis were compared between the 2 groups, as well as between IC subgroups with and without HL. Associations were evaluated after adjustment for potential confounders using regression models.

RESULTS: A total of 24,836 IC patients were identified and matched to 99,344 non-IC patients. Patients were predominantly female (92\%), with a mean age of $49.0(S D=15.3)$ years. IC patients used significantly more health care resources across all categories compared with non-IC patients. On average, having IC was associated with $\$ 7,223$ higher total health care costs than not having IC $(95 \% \mathrm{Cl}=\$ 6,650-\$ 7,796)$, with outpatient costs contributing to $71 \%$ of the difference, after adjusting for baseline age, gender, region, insurance type, plan type, and Charlson Comorbidity Index (CCI) score. The odds of developing select comorbidities were 2.61 times greater in IC patients compared with non-IC patients $(95 \% \mathrm{Cl}=2.52-2.70)$, adjusting for baseline age, sex, region, and CCI score. Among IC patients, the HL subgroup $(n=292)$ used more health care resources, and having $\mathrm{HL}$ was associated with $\$ 6,895$ higher total health care costs compared with not having $\mathrm{HL}(95 \% \mathrm{Cl}=\$ 3,770-\$ 10,020)$ after adjusting for baseline age, gender, region, insurance type, and plan type.

CONCLUSIONS: Findings suggest that patients with IC have significantly higher health care utilization, costs, and comorbidities compared with nonIC patients. This economic burden is further amplified in those with HL.

J Manag Care Spec Pharm. 2017;23(4):474-82

Copyright $\odot 2017$, Academy of Managed Care Pharmacy. All rights reserved.

\section{What is already known about this subject}

Without effective and lasting treatment options to manage their debilitating symptoms, interstitial cystitis (IC) patients experience a reduced quality of life and exert a significant economic burden. Based on 1998-2003 claims data from health plan-specific populations, IC patients have an annual health care utilization rate that is more than twice that of matched non-IC patients, as well as incur $\$ 3,000-\$ 4,000$ more in yearly health care costs.

IC patients with Hunner's lesions (HL) are known to experience a distinct clinical course, but their economic burden has never been characterized and compared with patients without HL.

\section{What this study adds}

This study compared health care utilization, costs, and comorbidities for IC patients to a matched cohort of non-IC patients using 2009-2014 claims data from a nationally representative population.

This study characterized and compared health care utilization, costs, and comorbidities for IC patients with HL to those without HL.

工 nterstitial cystitis (IC) is a chronic, debilitating condition that is characterized by persistent bladder pain, urinary urgency, frequent urination, and nocturia. Although known to predominantly occur in females, the etiology and pathophysiology of IC remain unclear, making the condition challenging to diagnose and to treat.1,2 Without clear diagnostic standards, it is common for IC patients to go undiagnosed for 5 years. ${ }^{3}$ Consequently, estimates of IC prevalence vary between $0.2 \%$ to $4.6 \%$ of the U.S. population, with some suggesting that the figure could be much higher. ${ }^{4-6}$ Additionally, approximately $10 \%-56 \%$ of IC patients also experience painful lesions on the bladder wall, known as Hunner's lesions (HL), formerly called Hunner's ulcers., ${ }^{7,8}$ While the presence of HL has been traditionally classified as a subtype of IC, recent research suggests that HL may represent its own distinct condition. ${ }^{8}$ Not only do IC patients with HL differ from those without HL demographically, but they also respond differently to existing treatments. ${ }^{9,10}$

In general, IC is treated with a variety of pharmacologic and medical interventions, most of which are off-label and all of which provide temporary, suboptimal relief. The only 
treatments approved by the U.S. Food and Drug Administration for IC are pentosan polysulfate sodium (Elmiron) and dimethyl sulfoxide (RIMSO-50). However, given conflicting evidence regarding the effectiveness of these treatments, current guidelines do not make any recommendations for using one agent over another to treat IC patients without HL. ${ }^{10}$ In contrast, guidelines recommend fulguration, a surgical procedure to remove lesions, to treat those patients with HL. ${ }^{10}$ Although fulguration is the best available approach to treating HL, it does not provide permanent relief so does not eliminate the need for additional pharmacologic and medical interventions. ${ }^{11}$

Without lasting treatment options, patients with IC experience a reduced quality of life and are expected to be a significant economic burden to society. To date, 4 claims-based analyses have been published on the economic burden of IC in the United States. However, these studies were conducted using older claims data that came from specific health plan types, which limit their generalizability. In 2006, Wu et al. published a retrospective claims-based analysis to describe the health care utilization, costs, and comorbidities associated with IC using 1999-2002 data from 16 large, self-insured U.S. companies. ${ }^{12}$ The authors found that IC patients incurred an average cost of $\$ 6,614$ in direct health care costs during the first year after diagnosis, which was $\$ 3,756$ more than nonIC patients. ${ }^{12}$ In 2008, Clemens et al. found that IC patients incurred an average cost of $\$ 7,100$, which was $\$ 4,106$ more than non-IC patients from 1998-2003 data in a managed care population. ${ }^{13}$ In the same year, Stanford et al. found IC patients to cost, on average, $\$ 9,186$, using 2000-2005 managed care claims data, but did not compare these findings with a control group. ${ }^{14}$ Finally, in 2011, Anger et al. used claims data from 1999 to characterize the costs specific to treating IC but did not capture the all-cause cost of having IC. ${ }^{15}$

Current literature falls short in fully capturing the extent of the economic burden of IC. Claims-based studies published to date are based on older data from IC populations covered by specific types of health plans. Furthermore, no studies have specifically examined patients with HL, whose burden of illness is expected to differ significantly from patients without HL. The primary objective of this study was to describe and compare health care utilization, direct costs, and comorbidities for patients with IC and those without IC during the first year after diagnosis. The secondary objective was to describe and compare health care resource utilization, direct costs, and comorbidities for IC patients with HL and without HL during the first year after diagnosis.

\section{Methods}

\section{Data Source}

Data were obtained from Truven Health MarketScan Research Commercial and Medicare Supplemental Claims Databases. The databases are large, nationally representative databases that contain Health Insurance Portability and Accountability Act (HIPAA) compliant, de-identified, patient-level, paid, and adjudicated claims information for over 143 million unique patients in the United States. ${ }^{16,17}$ The Commercial Claims database includes private-sector claims data from approximately 100 payers and contains more than 500 million claim records. ${ }^{16,17}$ The Medicare Supplemental Claims database contains claims data on Medicare recipients who subscribe to Medicare supplemental coverage through privately insured fee-for-service (FFS) or capitated health plans. ${ }^{16,17}$ Claims from 2009 to 2014 were analyzed for this study. This study did not meet the federal definition of "human subjects research" so was exempt from institutional review board review by the University of Washington Human Subjects Division.

\section{Cohort Identification}

IC Patients. Patients were identified as having an IC diagnosis if they had at least 1 International Classification of Diseases, Ninth Revision, Clinical Modification (ICD-9-CM) code of 595.1 (chronic interstitial cystitis) in the 2009-2014 claims files. The first ICD-9-CM code of 595.1 was considered the index diagnosis. To ensure that a patient's first ICD-9-CM code of 595.1 was reflective of an incident IC diagnosis, patients were excluded if they had any evidence of IC diagnosis or treatment 12 months before the date of their index diagnosis. Evidence of IC treatment included pharmacy claims for pentosan polysulfate sodium or medical claims for fulguration. Patients were further excluded if they were aged younger than 18 years; did not have 12 months of continuous enrollment pre- and postindex date; or had any bladder-related cancers (Figure 1). The 12 months previous to the index diagnosis date formed the pre-index or baseline period. The 12 months after the index diagnosis date formed the post-index or follow-up period.

Non-IC Patients. Four non-IC patients were randomly selected to match each IC patient on the basis of age, gender, and geographic region. Non-IC patients were required to not have an IC diagnosis or any evidence of IC treatment. Given that non-IC patients did not have a natural index diagnosis date, they were assigned an index date of the same month and year as their matched IC patients to ensure that the baseline and follow-up periods between the 2 cohorts were comparable. Non-IC patients were also excluded if they were aged younger than 18 years; did not have 12 months of continuous enrollment pre- and post-index diagnosis date of their matched case; or had any bladder-related cancers.

HL and Non-HL Subgroups. IC patients were further stratified into HL and non-HL subgroups. Because ICD-9-CM codes do not distinguish between IC with HL and without HL, fulguration was used as a proxy to identify IC patients with HL. To be considered part of the HL subgroup, IC patients were required to have at least 1 claim with a Current Procedural 


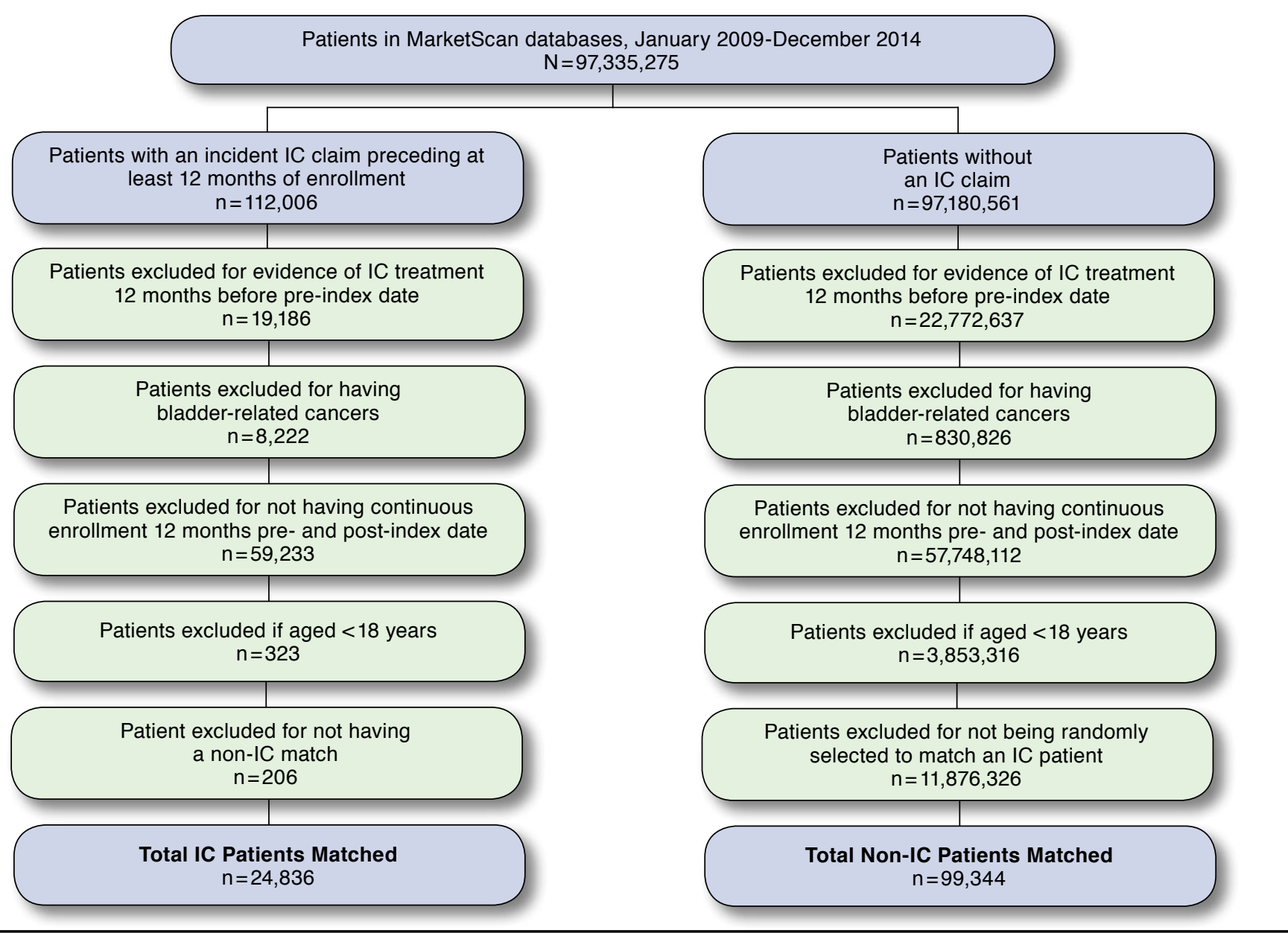

IC = interstitial cystitis

Terminology code for fulguration in the post-index period (see the Appendix, available in the online article).

\section{Assessment of Health Care Resource Utilization, Costs, and Comorbidities}

Health care resource utilization was determined by counting claims that occurred within the post-index period for inpatient admissions, outpatient visits, and emergency department (ED) visits. To assess meaningful prescription use, days supply for each prescription claim that occurred within the post-index period were summed. Total costs were adjusted to 2014 U.S. dollars using the Consumer Price Index and then summed for claims that occurred within the post-index period. For health care resource utilization and costs, mean counts and costs per patient, respectively, were reported for the following service categories: inpatient admissions, outpatient visits, ED visits, and prescription days supply. Additionally, costs associated with inpatient admissions, outpatient visits, and prescriptions filled were also summed to report a total health care cost. Incidences of select comorbidities with possible IC associations were also assessed. ${ }^{10}$ To be considered to have an incident comorbidity, patients were required to not have a claim associated with anxiety, depression, insomnia, vulvodynia, chronic fatigue syndrome, sicca syndrome, irritable bowel syndrome, migraines, or fibromyalgia during the pre-index period but to have a claim for the corresponding comorbidity during the post-index period. The proportion of patients with incident comorbidities was reported for all conditions collectively, as well as for each condition separately.

\section{Baseline Comorbidity}

To adjust for potential differences in baseline comorbidity that could affect health care use and cost during the post-index period, the Deyo-Charlson Comorbidity Index (CCI) score 


\begin{tabular}{|c|c|c|c|c|c|c|c|c|}
\hline \multirow[b]{2}{*}{ Age, mean (SD) } & \multicolumn{2}{|c|}{$\begin{array}{c}\text { IC } \\
(n=24,836)\end{array}$} & \multicolumn{2}{|c|}{$\begin{array}{c}\text { Non-IC } \\
(n=99,344)\end{array}$} & \multicolumn{2}{|c|}{$\begin{array}{c}\text { HL } \\
(n=292)\end{array}$} & \multicolumn{2}{|c|}{$\begin{array}{c}\text { Non-HL } \\
(n=24,544)\end{array}$} \\
\hline & 49.0 & (15.3) & 49.0 & $(15.3)$ & 50.8 & $(16.2)$ & 49.0 & $(16.2)$ \\
\hline \multicolumn{9}{|l|}{ Age group, years, \% (n) } \\
\hline$<31$ & 11.9 & $(2,961)$ & 11.9 & $(11,844)$ & 11.6 & $(34)$ & 11.9 & $(2,927)$ \\
\hline $31-40$ & 18.7 & $(4,642)$ & 18.7 & $(18,568)$ & 13.0 & $(38)$ & 18.8 & $(4,604)$ \\
\hline $41-50$ & 23.2 & $(5,763)$ & 23.2 & $(23,052)$ & 28.4 & $(83)$ & 23.1 & $(5,680)$ \\
\hline $51-60$ & 24.8 & $(6,148)$ & 24.8 & $(24,592)$ & 19.9 & $(58)$ & 24.8 & $(6,090)$ \\
\hline $61-70$ & 12.1 & $(3,012)$ & 12.1 & $(12,048)$ & 13.4 & (39) & 12.1 & $(2,973)$ \\
\hline $71-80$ & 6.4 & $(1,600)$ & 6.4 & $(6,400)$ & 8.6 & $(25)$ & 6.4 & $(1,575)$ \\
\hline$\geq 81$ & 2.9 & $(710)$ & 2.9 & $(2,840)$ & 5.1 & (15) & 2.8 & $(695)$ \\
\hline Female, \% (n) & 91.8 & $(22,793)$ & 91.8 & $(91,172)$ & 87.7 & $(256)$ & 91.8 & $(22,537)$ \\
\hline \multicolumn{9}{|l|}{ Region, \% (n) } \\
\hline North East & 11.8 & $(2,934)$ & 11.8 & $(11,736)$ & 15.4 & $(45)$ & 11.8 & $(2,889)$ \\
\hline North Central & 23.4 & $(5,816)$ & 23.4 & $(23,264)$ & 19.9 & (58) & 23.5 & $(5,758)$ \\
\hline South & 43.0 & $(10,675)$ & 43.0 & $(42,700)$ & 44.2 & (129) & 43.0 & $(10,546)$ \\
\hline West & 21.3 & $(5,297)$ & 21.3 & $(21,188)$ & 20.2 & (59) & 21.3 & $(5,238)$ \\
\hline Unknown & 0.5 & (114) & 0.5 & (456) & 0.3 & (1) & 0.5 & (113) \\
\hline \multicolumn{9}{|l|}{ Insurance type, \% (n) } \\
\hline Commercial & 82.2 & $(20,423)$ & 81.7 & $(81,148)$ & 77.1 & $(225)$ & 82.3 & $(20,198)$ \\
\hline Medicare Supplemental & 17.8 & $(4,413)$ & 16.7 & $(16,626)$ & 23.0 & (67) & 17.7 & $(4,346)$ \\
\hline Unknown & 0.0 & $(0)$ & 1.6 & $(1,570)$ & 0.0 & $(0)$ & 0.0 & $(0)$ \\
\hline \multicolumn{9}{|l|}{ Plan type, \% (n) } \\
\hline Comprehensive & 11.4 & $(2,837)$ & 10.6 & $(10,520)$ & 15.4 & $(41)$ & 11.4 & $(2,837)$ \\
\hline $\mathrm{HMO}^{\mathrm{a}}$ & 13.2 & $(3,274)$ & 15.6 & $(15,492)$ & 14.0 & $(164)$ & 13.2 & $(3,274)$ \\
\hline FFS $^{b}$ & 61.8 & $(15,354)$ & 58.6 & $(58,241)$ & 56.2 & (30) & 61.8 & $(15,354)$ \\
\hline CDHP and HDHP & 12.0 & $(2,986)$ & 12.0 & $(11,946)$ & 10.3 & $(292)$ & 12.0 & $(2,986)$ \\
\hline Unknown & 1.6 & $(385)$ & 3.2 & $(3,145)$ & 4.1 & (12) & 1.6 & (385) \\
\hline Charlson Comorbidity Index, mean (SD) & 0.58 & $(1.14)$ & 0.42 & $(1.03)$ & 0.72 & $(1.31)$ & 0.58 & $(1.14)$ \\
\hline \multicolumn{9}{|l|}{ Charlson Comorbidity Index, \% (n) } \\
\hline 0 & 70.5 & $(17,507)$ & 78.7 & $(78,189)$ & 66.8 & $(195)$ & 70.5 & $(17,312)$ \\
\hline 1 & 19.5 & $(4,850)$ & 14.3 & $(14,177)$ & 19.9 & $(58)$ & 19.5 & $(4,792)$ \\
\hline 2 & 7.1 & $(1,770)$ & 5.0 & $(4,952)$ & 8.2 & $(24)$ & 7.1 & $(1,746)$ \\
\hline $3+$ & 2.9 & (709) & 2.0 & $(2,026)$ & 5.1 & (15) & 2.8 & (694) \\
\hline Baseline costs, median (IQR), \$ & $\begin{array}{r}3 \\
(17,04 \\
\end{array}$ & $\begin{array}{l}830 \\
7-74,787) \\
\end{array}$ & $\begin{array}{r}1 \\
(4,93 \\
\end{array}$ & $\begin{array}{l}-300 \\
-36,833)\end{array}$ & $\begin{array}{r}4 \\
(26,81 \\
\end{array}$ & $\begin{array}{l}12 \\
22,363)\end{array}$ & $\begin{array}{r}3 \\
(16,9 \\
\end{array}$ & $\begin{array}{l}8,612 \\
8-74,417)\end{array}$ \\
\hline
\end{tabular}

was calculated for each patient using inpatient and outpatient claims data 365 days before their index date. The CCI score assigns weights to comorbidities such as liver disease, diabetes, AIDS, and cancer, which are then added together to create a summary indicator of baseline health status. ${ }^{18}$ A higher CCI score indicates greater baseline risk.

\section{Statistical Analyses}

Baseline characteristics and demographics of the study cohort were summarized using the mean and standard deviation (SD) for continuous variables and frequencies and proportions for categorical variables.
To assess the association of IC diagnosis with health care utilization, negative binomial regression models were used to separately model inpatient admission, outpatient visit, and ED visit counts. A linear regression was used to model prescription days supply. Models estimating health care utilization adjusted for baseline age, gender, geographic region, insurance type (commercial vs. Medicare Supplemental), insurance plan type, and CCI score.

Cost data were heavily right-skewed, with the majority of patients having little to no cost. ${ }^{19}$ Although it is common to analyze cost data using a generalized linear model (GLM) with a log link and gamma family, misspecification of the link and variance functions can result in bias and inefficiency in 


\begin{tabular}{|c|c|c|c|}
\hline & $\begin{array}{c}\text { IC } \\
(\mathbf{n}=24,836)\end{array}$ & $\begin{array}{c}\text { Non-IC } \\
(\mathrm{n}=99,344)\end{array}$ & $\begin{array}{c}\text { Adjusted Ratio } \\
\text { or Difference }^{\mathrm{a}}\end{array}$ \\
\hline & $\begin{array}{l}\text { Mean Number } \\
\text { (SD) }\end{array}$ & $\begin{array}{l}\text { Mean Number } \\
\text { (SD) }\end{array}$ & $\begin{array}{l}\text { Estimate } \\
(95 \% \text { CI })\end{array}$ \\
\hline $\begin{array}{l}\text { Inpatient } \\
\text { admissions }\end{array}$ & $0.15 \quad(0.52)$ & $0.09 \quad(0.36)$ & $\begin{array}{l}\text { IRR: } 1.54 \mathrm{~b} \\
(1.46-1.61)\end{array}$ \\
\hline Outpatient visits & $22.5 \quad(20.0)$ & $11.0 \quad(13.6)$ & $\begin{array}{l}\text { IRR: } 2.01^{\mathrm{b}} \\
(1.98-2.03)\end{array}$ \\
\hline ED visits & $0.45 \quad(1.49)$ & $0.19 \quad(0.69)$ & $\begin{array}{l}\text { IRR: } 2.25^{\mathrm{b}} \\
(2.15-2.35)\end{array}$ \\
\hline \multirow[t]{3}{*}{$\begin{array}{l}\text { Prescription } \\
\text { days supply }\end{array}$} & $1,079 \quad(1,071)$ & $657 \quad(857)$ & $\begin{array}{l}R D: 367 b \\
(356-377)\end{array}$ \\
\hline & $\begin{array}{c}\text { HL } \\
(\mathbf{n}=292)\end{array}$ & $\begin{array}{c}\text { Non-HL } \\
(\mathbf{n}=24,544)\end{array}$ & $\begin{array}{c}\text { Adjusted Ratio } \\
\text { or Difference }\end{array}$ \\
\hline & $\begin{array}{l}\text { Mean Number } \\
\text { (SD) }\end{array}$ & $\begin{array}{l}\text { Mean Number } \\
\text { (SD) }\end{array}$ & $\begin{array}{l}\text { Estimate } \\
(95 \% \mathrm{CI}) \\
\end{array}$ \\
\hline $\begin{array}{l}\text { Inpatient } \\
\text { admissions }\end{array}$ & $0.25 \quad(0.56)$ & $0.15 \quad(0.52)$ & $\begin{array}{l}\text { IRR: } 1.57^{\mathrm{b}} \\
(1.20-2.05)\end{array}$ \\
\hline Outpatient visits & $28.3 \quad(20.0)$ & $22.4 \quad(20.0)$ & $\begin{array}{l}\text { IRR: } 1.27^{\mathrm{b}} \\
\text { (1.17-1.38) }\end{array}$ \\
\hline ED visits & $0.60 \quad(1.19)$ & $0.45 \quad(1.49)$ & $\begin{array}{l}\text { IRR: } 1.32^{\mathrm{c}} \\
(1.04-1.67)\end{array}$ \\
\hline $\begin{array}{l}\text { Prescription } \\
\text { days supply }\end{array}$ & $1,238 \quad(1,133)$ & $1,077 \quad(1,070)$ & $\begin{array}{l}\text { RD: } 109 \\
(-3-220) \\
\end{array}$ \\
\hline \multicolumn{4}{|c|}{$\begin{array}{l}\text { aAdjusted for baseline age, gender, region, insurance type, plan type, and Cha } \\
\text { Comorbidity Index. } \\
b P \text { value } \leq 0.001 \text {. } \\
\text { CP value } \leq 0.05 \text {. } \\
C I=\text { confidence interval; } E D=\text { emergency department; HL=Hunner's lesions; } \\
\text { IC= interstitial cystitis; IRR=incidence rate ratio; } R D=\text { risk difference; } \\
S D=\text { standard deviation. }\end{array}$} \\
\hline
\end{tabular}

estimation. To overcome these issues, we modeled costs using an extended GLM approach ("pglm" command in STATA), which allows for estimation of flexible link and variance functions. ${ }^{20}$ We adjusted for baseline age, gender, geographic region, insurance type (commercial vs. Medicare Supplemental), insurance plan type, and CCI score. While baseline costs are often included as a covariate in cost models, we did not adjust for baseline costs. Given that IC is often diagnosed years after its manifestation, costs incurred during the 12 months before IC diagnosis were thought to reflect untreated IC rather than true baseline costs. In fact, the data confirmed this expectation, showing a distribution of baseline costs for IC patients that was much higher and overlapped minimally with the distribution of baseline costs for non-IC patients (Table 1).

Recycled predictions were used to estimate incremental costs of IC versus no IC among IC patients only (using the "pglmpredict" command in STATA). The model estimating incremental costs of HL also used an extended GLM and adjusted for baseline age, gender, geographic region, insurance type (commercial vs. Medicare Supplemental), and insurance plan type. As before, this model also did not adjust for baseline costs. Additionally, this model did not adjust for CCI because of convergence issues. Nevertheless, given that the distribution of CCI between HL and non-HL patients was similar in our sample, we did not expect bias due to confounding by comorbidities.

To model incident comorbidities, logistic regression models were fit for all comorbidities combined as well as for each comorbidity separately. All models adjusted for baseline age, gender, region, and CCI score.

All multivariable analyses used robust standard errors and assumed a significance level of $\alpha=0.05$. Analyses were conducted using SAS version 9.3 (SAS Institute, Cary, NC) and STATA 13-IC version 13.1 (StataCorp, College Station, TX).

\section{Results}

\section{Study Population}

A total of 24,836 IC patients were identified and matched to 99,344 non-IC patients on the basis of age, gender, and region. As shown in Table 1, IC patients and non-IC patients were mostly female (92\%), in the age range of $41-60$ years (48\%), and from the southern region of the United States (43\%). The majority of IC patients and non-IC patients had commercial health coverage $(83 \%)$ and subscribed to an FFS plan $(62 \%$ of IC patients, 59\% of non-IC patients). IC patients and non-IC patients were similar across all baseline characteristics with the exception of CCI score, with a greater proportion of IC patients having CCI scores greater than 1 when compared with non-IC patients (30\% vs. $21 \%$, respectively).

Of the IC patients, 292 (1.2\%) patients were identified as having HL. Non-HL and HL patients were similar in age (mean age $=49.0[\mathrm{SD}=15.3]$ vs. $50.8[\mathrm{SD}=16.2]$, respectively) and in proportion of CCI score greater than or equal to 1 (30\% vs. $33 \%$, respectively). HL and non-HL patients were predominantly female (92\% and 88\%, respectively).

\section{Health Care Utilization}

IC patients used significantly more health care resources across all service categories when compared with non-IC patients (Table 2). Of note, IC patients had twice as many outpatient and ED visits as did non-IC patients. On average, IC patients had 22.5 ( $S D=20.0)$ outpatient visits in the first 12 months after diagnosis, whereas their matched non-IC patients had 11.0 visits $(S D=13.6)$. Similarly, IC patients had an average of 0.45 ( $\mathrm{SD}=1.5$ ) ED visits in the first 12 months after diagnosis, whereas non-IC patients had 0.19 ED visits (SD=0.7). Furthermore, IC patients filled a prescription days supply of $1,079(\mathrm{SD}=1,071)$ compared with non-IC patients who filled 657 (SD = 857). Among the IC patients, HL patients used significantly more health care resources across all service categories compared with non-HL patients (Table 2). On average, HL patients had 6 more outpatient visits than non-HL patients in the first year after IC diagnosis (28.3 vs. 22.4 , respectively). 


\begin{tabular}{|c|c|c|c|}
\hline TABLE 3 & \multicolumn{2}{|c|}{ Total Health Care Costs } & \multirow[b]{2}{*}{$\begin{array}{c}\text { Adjusted } \\
\text { Incremental } \\
\text { Difference }^{\mathrm{a}}\end{array}$} \\
\hline & $\begin{array}{c}\text { IC } \\
(\mathbf{n}=24,836)\end{array}$ & $\begin{array}{c}\text { Non-IC } \\
(\mathrm{n}=99,344)\end{array}$ & \\
\hline & Mean (SD), \$ & Mean (SD), \$ & Estimate $(95 \% \mathrm{CI})$ \\
\hline Total & $15,711(28,093)$ & $7,393(21,420)$ & $7,223 \quad(6,650-7,796)$ \\
\hline $\begin{array}{l}\text { Inpatient } \\
\text { admissions }\end{array}$ & $2,872(17,110)$ & $1,755(13,414)$ & $841 \quad(468-1,214)$ \\
\hline Outpatient visits & $9,704(16,482)$ & $4,105(12,256)$ & $5,123 \quad(4,747-5,499)$ \\
\hline ED visits & $892 \quad(3,553)$ & $354 \quad(1,861)$ & $(375-649)$ \\
\hline \multirow[t]{3}{*}{ Prescriptions filled } & $3,113 \quad(6,746)$ & $1,528 \quad(4,154)$ & $1,403 \quad(1,307-1,499)$ \\
\hline & $\begin{array}{c}\text { HL } \\
(n=292)\end{array}$ & $\begin{array}{c}\text { Non-HL } \\
(\mathrm{n}=24,544)\end{array}$ & $\begin{array}{c}\text { Adjusted } \\
\text { Incremental } \\
\text { Difference }^{b} \\
\end{array}$ \\
\hline & Mean (SD), \$ & Mean (SD), $\$$ & Estimate $(95 \% \mathrm{CI})$ \\
\hline Total & $22,696(22,840)$ & $15,628(28,139)$ & $6,895(3,770-10,020)$ \\
\hline $\begin{array}{l}\text { Inpatient } \\
\text { admissions }\end{array}$ & $4,574(12,863)$ & $2,852(17,153)$ & $1,531(-4,068-7,130)$ \\
\hline Outpatient visits & $14,480(13,367)$ & $9,647(16,507)$ & $5,067 \quad(3,139-6,995)$ \\
\hline ED visits & $1,201 \quad(3,352)$ & $888 \quad(3,556)$ & $300 \quad(-708-1,308)$ \\
\hline Prescriptions filled & $3,627 \quad(5,456)$ & $3,107 \quad(6,760)$ & $(-158-892)$ \\
\hline \multicolumn{4}{|c|}{$\begin{array}{l}\text { "Adjusted for baseline age, gender, region, insurance type, plan type, and Charlson } \\
\text { Comorbidity Index. Significance must be obtained from confidence intervals, since } \\
\text { the "pglm" command in Stata does not return P values. } \\
\text { bAdjusted for baseline age, gender, region, insurance type, and plan type. } \\
\text { Significance must be obtained from confidence intervals (significant if confidence } \\
\text { interval does not include zero), since the "pglm" command in Stata does not return } \\
P \text { values. } \\
C I=\text { confidence interval; ED = emergency department; HL=Hunner's lesions; } \\
I C=\text { interstitial cystitis; SD=standard deviation. }\end{array}$} \\
\hline
\end{tabular}

After adjusting for baseline characteristics, IC patients used significantly more health care resources across all service categories when compared with non-IC patients (Table 2). IC patients had outpatient visits 2.01 times as often (95\% confidence interval $[\mathrm{CI}]=1.98-2.03, P<0.001$ ) and $\mathrm{ED}$ visits 2.25 times as often as non-IC patients $(95 \% \mathrm{CI}=2.15-2.35$, $P<0.001$ ). Additionally, after adjusting for baseline characteristics, IC patients filled 367 more prescription days supply than non-IC patients $(95 \% \mathrm{CI}=356-377, \mathrm{P}<0.001)$. Among IC patients, adjusted health care utilization was significantly higher in HL patients compared with non-HL patients across all service categories except prescription days supply. HL patients had inpatient admissions 1.57 times as often as nonHL patients (95\% CI $=1.20-2.05, P<0.001)$.

\section{Direct Costs}

IC patients had higher mean health care costs than non-IC patients across all service categories (Table 3). IC patients incurred more than double the total health care costs compared with non-IC patients $(\$ 15,711$ [SD $=\$ 28,093]$ vs. $\$ 7,393$ $[S D=\$ 21,420]$, respectively). Of the difference in total health care costs, outpatient visits accounted for the greatest portion

\section{TABLE 4 Incident Comorbidities}

\begin{tabular}{|c|c|c|c|c|}
\hline & \multicolumn{2}{|c|}{$\begin{array}{c}\text { IC } \\
(\mathrm{n}=24,836)\end{array}$} & $\begin{array}{c}\text { Non-IC } \\
(\mathrm{n}=99,344)\end{array}$ & Adjusted Ratio ${ }^{a}$ \\
\hline & \multicolumn{2}{|c|}{$\%(n)$} & $\%(n)$ & OR $(95 \% \mathrm{CI})$ \\
\hline Any comorbidity & 24.2 & $(6,008)$ & $10.7(10,650)$ & $2.61 \quad(2.52-2.70)^{b}$ \\
\hline Anxiety & 9.0 & $(1,882)$ & $4.0 \quad(3,675)$ & $(2.22-2.49)^{\mathrm{b}}$ \\
\hline Chronic fatigue & & (209) & $0.3 \quad(287)$ & $(2.38-3.41)^{\mathrm{b}}$ \\
\hline Depression & 5.2 & $(1,181)$ & $2.4 \quad(2,240)$ & $(2.07-2.39)^{\mathrm{b}}$ \\
\hline ibromyalgia & 6.4 & $(1,433)$ & $2.1 \quad(2,030)$ & $(2.90-3.34)^{\mathrm{b}}$ \\
\hline nsomnia & 3.4 & $(804)$ & $1.5 \quad(1,493)$ & $(2.03-2.42)^{\mathrm{b}}$ \\
\hline BS & 3.6 & $(843)$ & $(751)$ & $(4.34-5.30)^{\mathrm{b}}$ \\
\hline Migraine headaches & 4.2 & $(956)$ & $(1,729)$ & $(2.20-2.59)^{\mathrm{b}}$ \\
\hline Sicca syndrome & 0.3 & (85) & (95) & $3.43 \quad(2.56-4.60)$ \\
\hline \multirow[t]{3}{*}{ Vulvodynia } & 0.9 & $(212)$ & (34) & $25.69(17.88-36.93)^{b}$ \\
\hline & \multicolumn{2}{|c|}{$\begin{array}{c}\text { HL } \\
(n=292)\end{array}$} & $\begin{array}{c}\text { Non-HL } \\
(\mathrm{n}=24,544)\end{array}$ & Adjusted Ratio $^{a}$ \\
\hline & \multicolumn{2}{|c|}{$\%(n)$} & $\%(n)$ & OR $(95 \%$ CI) \\
\hline Any comorbidity & 22.6 & (66) & $24.2 \quad(5,942)$ & $0.95 \quad(0.72-1.25)$ \\
\hline Anxiety & 7.1 & (17) & $9.0 \quad(1,865)$ & $(0.49-1.32)$ \\
\hline Chronic fatigue & 0.4 & (3) & (206) & $(0.39-3.93)$ \\
\hline Depression & 7.2 & (19) & $(1,162)$ & $(0.92-2.35)$ \\
\hline ibromyalgia & 5.2 & (14) & $(1,419)$ & $(0.48-1.42)$ \\
\hline nsomnia & 2.5 & $(7)$ & $(797)$ & $(0.35-1.58)$ \\
\hline BS & 4.7 & (13) & $(830)$ & $(0.77-2.39)$ \\
\hline Migraine headaches & 2.3 & $(6)$ & $(950)$ & $(0.25-1.25)$ \\
\hline icca syndrome & 0.3 & (1) & (84) & $(0.13-7.08)$ \\
\hline Vulvodynia & 0.7 & (2) & $(210)$ & $(0.21-3.42)$ \\
\hline
\end{tabular}

${ }^{a}$ Adjusted for baseline age, sex, region, and Charlson Comorbidity Index.

${ }^{b} P$ value $\leq 0.001$.

$C I=$ confidence interval; $H L=$ Hunner's lesions; $I B S=$ irritable bowel syndrome; $I C=$ interstitial cystitis; $O R=$ odds ratio.

with IC patients spending 3 times the amount as non-IC patients $(\$ 9,704[\mathrm{SD}=\$ 16,482]$ vs. $\$ 4,105[\mathrm{SD}=\$ 12,256]$, respectively). After outpatient visits, prescription costs accounted for the next greatest difference in total health care costs, with IC patients spending twice the amount as non-IC patients $(\$ 3,113$ $[\mathrm{SD}=\$ 6,746]$ vs. $\$ 1,528$ [SD $=\$ 4,154]$, respectively).

After adjusting for baseline characteristics, having IC was associated with significantly higher costs than not having IC across all service categories (Table 3). Having IC was associated with $\$ 7,223$ more in total health care costs than not having IC (95\% CI $=\$ 6,650-\$ 7,796)$. Outpatient visits accounted for the greatest portion $(71 \%)$ of this difference, with having IC associated with $\$ 5,123$ more on outpatient visits (95\% $\mathrm{CI}=\$ 4,747-\$ 5,499)$. Prescriptions accounted for the second greatest portion (19\%) of the total health care cost difference, with having IC associated with $\$ 1,403$ more on prescriptions than not having IC $(95 \% \mathrm{CI}=\$ 1,307-\$ 1,499)$.

HL patients had higher mean costs than non-HL patients in the total, inpatient admissions, and outpatient visits service categories. On average, HL patients spent nearly 50\% more in total costs compared with non-HL patients $(\$ 22,696$ 
[SD $=\$ 22,840]$ vs. $\$ 15,628$ [SD $=\$ 28,139]$, respectively). Of the difference in total health care costs, outpatient visits accounted for the greatest portion, with IC patients spending $\$ 14,480(\mathrm{SD}=\$ 13,367)$ and non-IC patients spending $\$ 9,647$ $(\mathrm{SD}=\$ 16,507)$.

After adjusting for baseline characteristics, having HL was associated with significantly more total and outpatient visit costs compared with not having HL. Having HL was associated with $\$ 6,895$ more in total costs than not having HL patients (95\% CI $=\$ 3,770-\$ 10,020)$. Again, outpatient visits accounted for the greatest portion of this difference, with having HL associated with $\$ 5,067$ more on outpatient visits than not having HL (95\% CI $=\$ 3,139-\$ 6,995)$. Inpatient admissions accounted for the second greatest portion of the total difference, with having HL associated with $\$ 1,531$ more than not having HL, although this difference was not statistically significant (95\% CI $=-\$ 4,068-\$ 7,130)$.

\section{Incident Comorbidities}

As shown in Table 4, the proportion of IC patients who developed comorbidities was greater than the proportion of non-IC patients across all comorbidities selected for assessment. The proportion of IC patients who developed any comorbidity was more than twice the proportion of non-IC patients ( $24 \%$ vs. $11 \%$, respectively). The most common comorbidity developed was anxiety ( $9 \%$ vs. $4 \%$, respectively), followed by fibromyalgia ( $6 \%$ vs. $2 \%$, respectively) and depression ( $5 \%$ vs. $2 \%$, respectively). The proportions of HL patients who developed comorbidities were similar to non-HL patients across all comorbidities assessed. The proportion of HL patients who developed any comorbidity was similar to non-HL patients ( $23 \%$ vs. $24 \%$, respectively).

After adjusting for baseline characteristics, the odds of developing comorbidities was significantly greater among IC patients than non-IC patients across all comorbidities assessed. IC patients had 2.61 times higher odds of developing a comorbidity (95\% CI $=2.52-2.70, P<0.001$ ). IC patients had more than 3 times higher odds of developing vulvodynia (odds ratio $[O R]=25.69 ; 95 \% C I=17.88-36.93 ; P<0.001)$, irritable bowel syndrome (IBS; OR=4.80; 95\% CI =4.34-5.30; $P<0.001$ ), sicca syndrome $(\mathrm{OR}=3.43 ; 95 \% \mathrm{CI}=2.56-4.60 ; \mathrm{P}<0.001)$, and fibromyalgia $(\mathrm{OR}=3.11 ; 95 \% \mathrm{CI}=2.90-3.34 ; \mathrm{P}<0.001)$ than non-IC patients. After adjusting for baseline characteristics, the odds of developing comorbidities were not found to be significantly different between HL and non-HL patients across all comorbidities assessed (Table 4).

\section{Discussion}

This study characterized and compared health care resource utilization, costs, and comorbidities between IC and non-IC patients and, among IC patients, between patients with and without HL. Similar to previously published claims studies, this study found that IC patients used significantly more health care resources, incurred more costs, and developed more comorbidities than non-IC patients in the first 12 months after diagnosis.

As for health care resource utilization, this study found IC patients to have, on average, 0.15 inpatient admissions during the first 12 months after diagnosis, which was similar to Clemens et al, who found an average of 0.17 inpatient admissions. ${ }^{13}$ In contrast, this study found IC patients to have, on average, 22.5 outpatient visits during the first 12 months after diagnosis, which was higher than the 14.1 outpatient visits found in Clemens et al. ${ }^{13}$ The difference in outpatient visits could be because Clemens et al. estimated health care resource utilization in 1 managed care population, which could have had different practice patterns than the population of our study, which was composed of patients from different types of health plans.

With respect to costs, our study found the mean total cost of IC to be $\$ 15,711$. After adjusting for baseline characteristics, the incremental cost of IC was $\$ 7,223$ greater than the cost of not having IC. This study also found that the difference in total health care cost between having and not having IC was primarily driven by differences in outpatient visits costs, which accounted for $71 \%$ of the difference in total health care costs. In 2006, Wu et al. found IC patients to cost, on average, $\$ 6,614$ (2002 U.S. dollars) in direct costs during the first year after diagnosis, which after adjusting for confounders, was $\$ 2,309$ more than non-IC patients. ${ }^{12}$ Additionally, they found that outpatient visits accounted for $51 \%$ of the difference in total health care costs between IC and non-IC patients.

One reason that our study may have found absolute value costs that were higher than $\mathrm{Wu}$ et al. is that only payer costs were included, whereas our study included payer costs as well as patient costs (i.e., copay, deductible, and coinsurance costs). Another reason for this difference is that $\mathrm{Wu}$ et al. only examined the costs that incurred over 48 weeks after index diagnosis, whereas our study examined costs that incurred over 52 weeks after index diagnosis, but this would not have contributed largely to this difference. The mean total cost of IC found in this study was also higher than the \$9,186 (2005 U.S. dollars) that Stanford et al. found IC patients to spend during the first year after diagnosis, of which 56\% were a result of outpatient visits. ${ }^{14}$ Similar to Clemens et al., Stanford's analysis was based on data from all managed care plans, which could collectively have lower costs than our study, which was, again, based on data from a variety of health plan types.

In another similiarity to our study, Wu et al. found IC to be associated with higher risks for certain comorbidities..$^{12}$ They found relative risks for IBS, depression, and anxiety to be 4.9, 2.8 , and 4.5 , respectively, whereas we found adjusted odds 
ratios of 4.8, 2.2, and 2.4, respectively. One reason that Wu et al.'s estimates may have been higher is that they were based on prevalent comorbidities, whereas ours were based on incident comorbidities. Also, Wu et al.'s estimates were not adjusted for potential confounders, whereas ours were.

In addition, our study found that among IC patients, those with HL had a higher utilization of health care resources across all service categories except for prescription days supply filled, when compared with non-HL patients. In terms of costs, having HL was associated with significantly higher total and outpatient costs. Since no differences were found between HL and non-HL patients in terms of incident comorbidities, the higher health care resource utilization in HL patients could be related to $\mathrm{HL}$ patients requiring a more intensive treatment of IC. The biggest difference in health care resource utilization was because of outpatient visits. One reason why HL patients could have had more outpatient visits is because they were identified by using fulguration as a proxy, and fulguration is primarily an outpatient procedure.

\section{Limitations}

This study has several limitations. As with other claims analyses, this study was subject to potential coding error, which could lead to misclassification of exposure and outcomes variables. Relying on ICD-9-CM codes to identify IC diagnosis could lead to misclassification of those who really do have IC if the ICD-9-CM code for IC was billed for the purposes of differential diagnosis. Additionally, as with other claims analyses, this study is subject to healthy-worker bias, since it inherently requires that patients or their dependents are healthy enough to work and receive health insurance. This bias would cause health care utilization, costs, and comorbidities to be higher than what was observed in this study. Furthermore, given that the Truven Health MarketScan Research Databases have greater representation from the southern region of the United States, findings of this study could be skewed to reflect demographics or practice patterns from that region.

Next, this study used fulguration as a proxy for patients with HL, which could lead to misclassification of the exposure, given that patients with HL may not have received fulguration within the first year after diagnosis. As a result, some patients with HL could have been misclassified as patients without HL, leading to an attenuation of the difference between HL and non-HL patients. Using fulguration as a proxy for HL patients could also lead to an overestimate of resource utilization and costs, since the procedure of fulguration is associated with office visits and costs. Finally, the association between IC and having higher odds of incident comorbidities may be a result, in part, of IC patients having more opportunity to receive such diagnoses, since they are more frequently coming into contact with health care providers for treatment of IC.
This study's strengths include a large sample size, as well as being the first study to characterize and compare differences between IC patients with and without HL. Also, it is the first study to our knowledge that uses an extended GLM to estimate and compare adjusted incremental costs of IC. In addition, it is the first study to capture costs from the payer and patient perspectives, as well as reflect costs of a variety of health plan types. This study is also the first study to provide adjusted health care resource utilization and incident comorbidities.

\section{Conclusions}

The results of this study indicate that IC is associated with significant health care utilization, direct costs, and comorbidities in the first year after diagnosis. In general, this burden was even higher in the presence of HL. Future work allowing for a longer-term follow-up is warranted not only to more accurately capture those with HL but also to better understand the longterm burden of IC. Since IC is not well controlled by traditional pharmacologic agents, patients may resort to more invasive and expensive interventions in the long run.

\section{Authors}

AMY TUNG, PharmD, MS, and AASTHAA BANSAL, PhD, Pharmaceutical Outcomes Research and Policy Program, University of Washington, Seattle. ZSOLT HEPP, PharmD, MS, Allergan, Irvine, California, and EMILY BETH DEVINE, PhD, PharmD, MBA, Pharmaceutical Outcomes Research and Policy Program; Department of Health Services; and Department of Surgery, University of Washington, Seattle.

AUTHOR CORRESPONDENCE: Emily Beth Devine, PhD, PharmD, MBA, University of Washington, 1959 N.E. Pacific St., H375-D Health Sciences Bldg., Seattle, WA 98195. Tel.: 206.221.5760; E-mail:bdevine@uw.edu.

\section{DISCLOSURES}

Funding for this study was contributed by Allergan. Tung was supported by a training grant from Allergan at the time of this study. Hepp was an Allergan employee at the time this study was conducted. The other authors have nothing to disclose.

This research was previously presented, in part, as a poster presentation at the International Society for Pharmacoeconomics and Outcomes Research 21st Annual International Meeting; Washington, DC; May 23, 2016

Study concept and design were primarily contributed by Hepp, along with Tung and Devine. Tung took the lead in data collection, with assistance from Hepp, and data interpretation was performed by Tung, along with Bansal and Devine. The manuscript was prepared primarily by Tung, along with Devine, Bansal, and Hepp.

\section{REFERENCES}

1. Koziol JA, Clark DC, Gittes RF, et al. The natural history of interstitial cystitis: a survey of 374 patients. J Urol. 1993;149(3):465-69. 
2. Koziol JA. Epidemiology of interstitial cystitis. Urol Clin North Am. $1994 ; 21(1): 7-20$.

3. Chung MK, Jarnagin B. Early identification of interstitial cystitis may avoid unnecessary hysterectomy. JSLS. 2009;13(3):350-57.

4. Clemens JQ, Meenan RT, Rosetti MC, et al. Prevalence and incidence of interstitial cystitis in a managed care population. J Urol. 2005;173(1):98-102.

5. Suskind AM, Berry SH, Ewing BA, Elliott MN, Suttorp MJ, Clemens JQ. The prevalence and overlap of interstitial cystitis/bladder pain syndrome and chronic prostatitis/chronic pelvic pain syndrome in men: results of the RAND Interstitial Cystitis Epidemiology (RICE) Male Study. J Urol. 2013;189(1):141-45. Available at: https://www.ncbi.nlm.nih.gov/pmc/articles/ PMC3894747/. Accessed March 2, 2017.

6. Berry SH, Elliott MN, Suttorp M, et al. Prevalence of symptoms of bladder pain syndrome/interstitial cystitis among adult females in the United States. J Urol. 2011;186(2):540-44. Available at: https://www.ncbi.nlm.nih.gov/pmc/ articles/PMC3513327/. Accessed March 2, 2017.

7. Metts J. Interstitial cystitis: urgency and frequency syndrome. Am Fam Physician. 2001;64(7):1199-206.

8. Peeker R, Fall M. Towards a precise definition of interstitial cystitis: further evidence of differences in classic and nonulcer disease. J Urol. 2002;167(6):2470-72.

9. International Society for the Study of BPS. Hunner lesion (formerly known as Hunner's ulcer). Available at: http://www.essic.eu/hunner_lesion. html. Accessed March 2, 2017.

10. Hanno PM, Burks DA, Clemens JQ, et al. American Urological Association (AUA) Guideline. Diagnosis and treatment of interstitial cystitis/ bladder pain syndrome. 2014. Available at: https://www.auanet.org/common/pdf/education/clinical-guidance/IC-Bladder-Pain-Syndrome-Revised. pdf. Accessed March 2, 2017.
11. Ryu J, Pak S, Song M, Chun JY, Hong S, Choo MS. Elimination of Hunner's ulcers by fulguration in patients with interstitial cystitis: is it effective and long lasting? Korean J Urol. 2013;54(11):767-71.

12. Wu EQ, Birnbaum H, Mareva M, et al. Interstitial cystitis: cost, treatment and comorbidities in an employed population. Pharmacoeconomics. 2006;24(1):55-65.

13. Clemens JQ, Meenan RT, O'Keeffe Rosetti MC, Kimes T, Calhoun EA. Costs of interstital cystitis in a managed care population. Urology. 2008;71(5):776-80. Available at: https://www.ncbi.nlm.nih.gov/pmc/articles/ PMC2429850/. Accessed March 2, 2017.

14. Stanford EJ, Chen A, Wan GJ, et al. Treatment modalities, health care resource utilization, and costs in patients diagnosed with interstitial cystitis. Am J Obstet Gynecol. 2008;199(1):71.el-10.

15. Anger JT, Zabihi N, Clemens JQ, Payne CK, Saigal CS, Rodriguez LV. Treatment choice, duration, and cost in patients with interstitial cystitis and painful bladder syndrome. Int Urogynecol J. 2011;22(4):395-400.

16. Hansen LG, Chang S. Health research data for the real world: the MarketScan databases. Truven Health Analytics white paper. July 2011. Available at: http://truvenhealth.com/portals/0/assets/PH_11238_0612_ TEMP_MarketScan_WP_FINAL.pdf. Accessed March 2, 2017.

17. Truven Health Analytics. MarketScan Commercial Claims and Encounters Medicare Supplemental User Guide: Data Year 2011 Edition. Ann Arbor, MI: Truven Health Analytics; 2012.

18. Deyo RA, Cherkin DC, Ciol MA. Adapting a clinical comorbidity index for use with ICD-9-CM administrative databases. J Clin Epidemiol. 1992;45(6):613-19.

19. Dodd S, Bassi A, Bodger K, Williamson P. A comparison of multivariable regression models to analyze cost data. J Eval Clin Pract. 2006;12(1):76-86.

20. Basu A. Extended generalized linear models: simultaneous estimation of flexible link and variance functions. Stata J. 2005;5(4):501. 


\section{APPENDIX ICD-9-CM and CPT Codes and NDC Numbers}

Condition

Interstitial cystitis

Fulguration

Charlson Comorbidity Index

Myocardial infarction

Congestive heart failure

Peripheral vascular disease

Cardiovascular disease

Chronic obstructive pulmonary disease

Dementia

Rheumatic disease

Peptic ulcer disease

Liver disease

Liver disease, complicated

Diabetes, no complications

Diabetes, complications

Paralysis

Renal disease

Cancer

Metastatic cancer

AIDS

\section{Comorbidities}

Anxiety

Depression

Migraine headache

Fibromyalgia

Irritable bowel syndrome

Vulvodynia

Chronic fatigue syndrome

Sicca syndrome

Insomnia

\section{Exclusion codes}

Pentosan polysulfate sodium

Bladder neoplasm

Carcinoma in situ of bladder

Malignant neoplasm, bladder neck

Malignant neoplasm, prostate

Malignant neoplasm, urethra

Malignant neoplasm, uterus, ovaries, fallopian tubes, adnexa,

\& unspecified ovary related

Radiation cystitis

Secondary malignant neoplasm, genital organs

Secondary urinary organ neoplasm

Spinal cord injury

Tuberculous cystitis

Unspecified bladder neoplasm

Unspecified neoplasm, genital organs

Urethral diverticulum

Urethral stricture

Vaginal cancer

AIDS = acquired immunodeficiency syndrome; CPT = Current Procedural Terminology; ICD-9-CM = International Classification of Diseases, Ninth Revision, Clinical

Modification; NDC= National Drug Code.
Codes

ICD-9-CM 595.1

CPT 52214, 52224

ICD-9-CM 410, 412

ICD-9-CM 428

ICD-9-CM441, 443.9, 785.4, V424

ICD-9-CM 430, 431, 432, 433, 434, 435, 436, 437, 438

ICD-9-CM 490, 491, 493, 494, 495, 496, 500, 510, 502, 503, 504, 505, 506.4

ICD-9-CM 290

ICD-9-CM 725, 710.0, 710.1, 710.4, 714.0, 714.1, 714.2

ICD-9-CM 531,532,533,534

ICD-9-CM 571.2, 571.4, 571.5, 571.6

ICD-9-CM 572.2, 456.0, 456.1, 456.20, 456.21

ICD-9-CM 250.00, 250.01, 250.02, 250.03, 250.07

ICD-9-CM 250.04, 250.05, 250.06

ICD-9-CM 342, 244.1

ICD-9-CM 582, 583, 585, 586, 588

ICD-9-CM 148-195, 200-208

ICD-9-CM 196, 197, 198

ICD-9-CM 042, 043, 044

ICD-9-CM 300.00, 300.01, 300.02

ICD-9-CM 311

ICD-9-CM 346

ICD-9-CM 564.1

ICD-9-CM 625.7

ICD-9-CM 780.71

ICD-9-CM 710.2

ICD-9-CM 780.52

NDC 00062980001, 00575760001, 17314930001, 50458009801, 54569512300,

$54569512301,54868452500,54868452501,54868452502,54868452503,68115088800$

ICD-9-CM 188.0-188.9

ICD-9-CM 233.7

ICD-9-CM 188.5

ICD-9-CM 185

ICD-9-CM 189.3

ICD-9-CM 179.*, 180.*, 180.1, 180.8, 180.9, 182, 182.1, 182.8, 180.0, 183.2-183.5, 183.8,

183.9

ICD-9-CM 595.82

ICD-9-CM 198.82

ICD-9-CM 198.1

ICD-9-CM 952.00-952.09, 952.10-952.19, 952.2, 952.3, 952.8-952.10

ICD-9-CM 16.10

ICD-9-CM 239.4

ICD-9-CM

ICD-9-CM 599.20

ICD-9-CM 598.0, 598.01, 598.1, 598.2, 598.8, 598.9

ICD-9-CM 184.00
ICD-9-CM 729.1 